\title{
Weakening and Deficiency: Environmental Justice in the Process of Urbanization in China
}

\author{
Ai Lijuan Wang Ziyan Du Chuntao \\ Department of Humanities and Social Sciences \\ Dalian University of Technology 116024
}

\begin{abstract}
Since the reform and opening up, the speed of urbanization in China has been accelerating and the environmental situation has become increasingly severe. In the process of accelerating urbanization, central cities have put forward the goal of building eco-cities, carrying out the second modernization, polluting enterprises gradually move from the central cities to the surrounding areas, a large amount of agricultural land has been requisitioned into nonagricultural land, and rural and wilderness areas have been gradually reduced. Due to various reasons, there are various environmental injustices in the three dimensions between man and nature, man and non-man species, and man and man. The ecological system is increasingly fragile, species diversity is reduced, the distribution of environmental goods and evils is unfair or the environmental value is difficult to effectively identify, resulting in frequent environmental conflicts and environmental crises, which seriously affects social stability and harmonious development. To construct the system of environmental justice in the process of urbanization, it is necessary to distribute environmental infrastructure and environmental responsibility equitably; improve the environmental legal system; coordinate environmental plannings and environmental development goals; carry out technological ecological transformation and governance of traditional industries; further cultivate social culture of environmental justice.
\end{abstract}

Keywords: Urbanization; Environmental justice; Environmental value; Environmental responsibility; Environmental conflict

The level of urbanization reflects the level of a country's economic development. With the continuous improvement of China's productivity and accelerated economic development, the urban population is rapidly expanding and the urbanization process has entered a new stage. According to the data released by State Statistics Bureau, China's urbanization rate has reached $49.68 \%$ in 2010, and the China's New-urbanization Report pointed out that China's urbanization rate exceeded $50 \%$ for the first time in 2011, which means that the urban population exceeded the rural population for the first time, and China's urbanization development has entered a critical stage. Urbanization has become an important measure for China to promote industrialization, solve employment, and expand domestic demand. At present, China's urbanization process and productivity development are largely driven by the development of the secondary industry, which is a development that despises environmental problems,trades resources for economic development, and ignores and destroys people's lives and the sustainable development of future generations.

Environmental justice is also called environmental equity.The content of environmental justice mainly includes three aspects: distributive justice, acknowledge justice and procedural justice, that is, all people enjoy the benefits brought by the environment and bear the corresponding environmental responsibility, acknowledge the existence of injustice, and make use of sound laws and regulations to make people have more equal participation rights to ensure the pursuit of environmental justice.In terms of distributive justice, which has been studied most at the present stage, it basically includes two aspects: first, in terms of the social relationship between people, everyone enjoys environmental rights fairly and assumes the responsibility of protecting the environment fairly; second, in terms of the ecological relationship between man and nature, all people should assumes the responsibility of compensating nature while developing and utilizing environmental resources to achieve a balance between utilization and protection.

From the perspective of the development of environmental ethics, environmental justice can also be understood from three levels: first, human beings should maintain the balance of the whole earth's ecosystem; second, human beings should treat non-human species fairly, protect rare species, and do not abuse animals andover-consume animals and plants; third, we need to share environmental rights and responsibilities equally among human beings, including within the same generation, between different generations, between different countries, ethnic groups, regions, strata and genders.

\section{The environmental effects of urbanization}

1.1 Environmental positiveeffects of urbanization 
In the process of urbanization in China, the construction of eco-city has become the goal of several central cities. Many cities have proposed the construction of ecological cities. Such as, Changsha proposed to build an eco-economic city, Jiangxi proposed to build an eco-economic zone, and Yunnan to build a green economy province. At present, there are already Dalian, Shenzhen, Xiamen, Weihai, Zhuhai, Zhangjiagang, Kunshan, Yantai, Laizhou, Zhongshan and other cities have formulated plans for the construction of eco-cities and carried out the practice of eco-cities construction.Shanghai has proposed the goal of "building an international eco-city in 2015" in 15 years.

Although the construction of eco-cities is not satisfactory, in the above cities, especially the central cities, have significantly improved their living environment indicators in terms of per capita housing, per capita green space, traditional environmental pollution control and other aspects.And these cities have taken on a new look and the quality of life of urban residents has been improved. All kinds of urban environmental governance systems are increasingly improving, and the basic urban and rural ecological public service system is under construction.

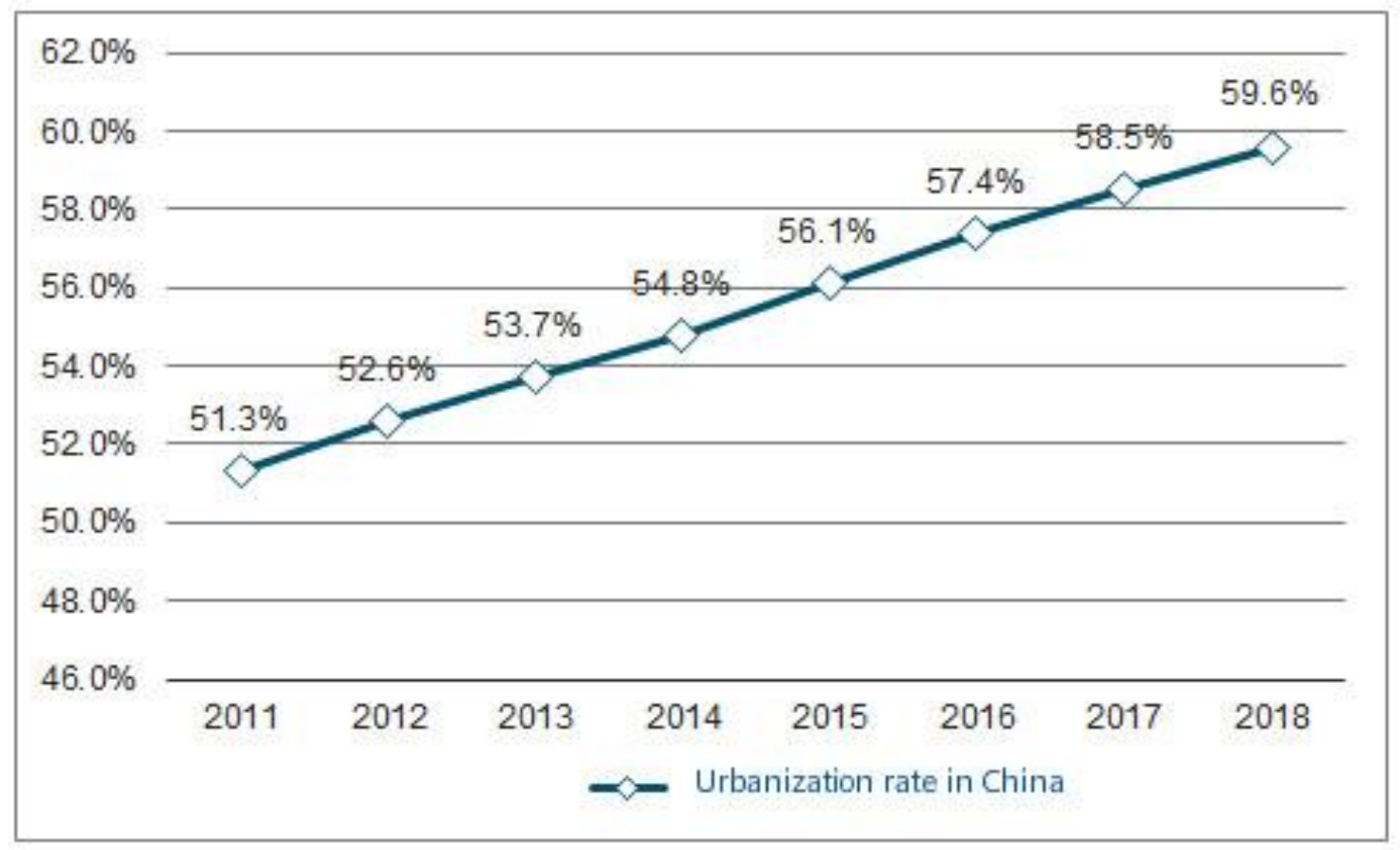

Figure 1 China's urbanization rate (2011-2018)The data come from the National Bureau of Statistics, PRC.

1.2 Environmental negative effects of urbanization

In the process of urbanization, urban space keeps expanding, large areas of agricultural land is converted into nonagricultural land, and the original pastoral and rural areas are continuously reduced and disappeared, which are incorporated into municipal planning and become urban built-up areas.In the process of land acquisition and demolition and old city reconstruction, the original historical and cultural landscape disappeared, and the historical buildings preserved for many years are demolished, so that future generations could no longer face the most vivid history. For example, the quadrangle courtyard in Beijing,and many former residences of celebrities; and the Japanese room in Dalian. It is reported that 80 villages have disappeared every year in China in the past 10 years.

With the chain reactions of urbanization, the wilderness is constantly being destroyed and disappeared.Taking wetlands as an example, China has joined the Convention onWetlands for 16 years, which is the 16-year-old wetlands protection intensity. A considerable number of wetlands have been rescued, but it is still difficult to stop the destruction of wetlands.According to satellite remote sensing data in the past 20 years, the total wetlands area in China has decreased from 366,000 square kilometers in 1990 to 324,000 square kilometers in 2008, a decrease of $11.46 \%$.If you trace back to the middle of the last century, the reduction in wetlands area is even more alarming.As a result, the habitat and breeding space of a large number of wild animals has been compressed, even they have no place to live. The disappearance of birds and the destruction of the natural environment are only superficial effects. Wetlands are very complex ecosystems, and even scientists cannot predict what kind of disasters may happen in the future if they are destroyed.Even if the biological chain is destroyed, it may have a profound impact on the genes of several generations in a region. 
Since the 1950s, the national wetland reclamation area has reached 10 million hectares, and the area along the beach has been cut by more than half. The original swamp in the Sanjiang Plain of Heilongjiang province has been cut by nearly $80 \%$, and the lakes in Hubei province, a province with thousands of lakes, have been reduced by two-thirds. And more than $56 \%$ of the mangroves are lost. Thousands of lakes of various sizes have disappeared in the country. The water quality of many wetlands has deteriorated year by year. Many wetland organisms are on the verge of extinction. About one-third of the natural wetlands are in danger of being changed and lost. China's existing natural or seminatural wetlands account for only $3.77 \%$ of its land area, far below the world average of $6 \%$, and the trend of area decline has not been effectively contained.

Under the guidance of eco-city construction and industrial gradient transfer, all major cities across the country have basically implemented relocation of industrial pollutionand transferred the polluting industries to surrounding areas. Since the 1990s, Guangdong province has moved a large number of polluting industries to other areas in the economically backward provinces. These areas have continuously accepted pollution projects from the Pearl River Delta Economic Zone from industrial transfer, and environmental pollution and deterioration in some areas has become increasingly serious. In large and medium-sized cities, the 15 types of heavily polluting small businesses that the state has banned have gradually disappeared,and a large part of them have migrated to the east and west wings of the relatively backward economy and the northern part of Guangdong. Taking Beijing as an example, all polluting enterprises will be relocated and settled in the urban-rural fringe and rural areas outside the Fifth Ring Road. This will greatly promote the industrial upgrading of large cities and promote rural industrialization and urbanization. However, from the current point of view, due to the lack of appropriate pollution control technology and investment, this practice will inevitably aggravate rural environmental pollution while reducing urban environmental pollution.

Under the current development model, the transfer of high-risk and high-pollution industries from central cities to small and medium-sized cities has gradually become more common.In order to develop the economy, protect the environment and the interests of the people, the central cities use the construction of the metropolitan area to transfer pollution to small and medium-sized cities on the edge. The high-risk, high-pollution enterprises after the transfer have caused great harm to the local environment, which has led to a series of conflicts.

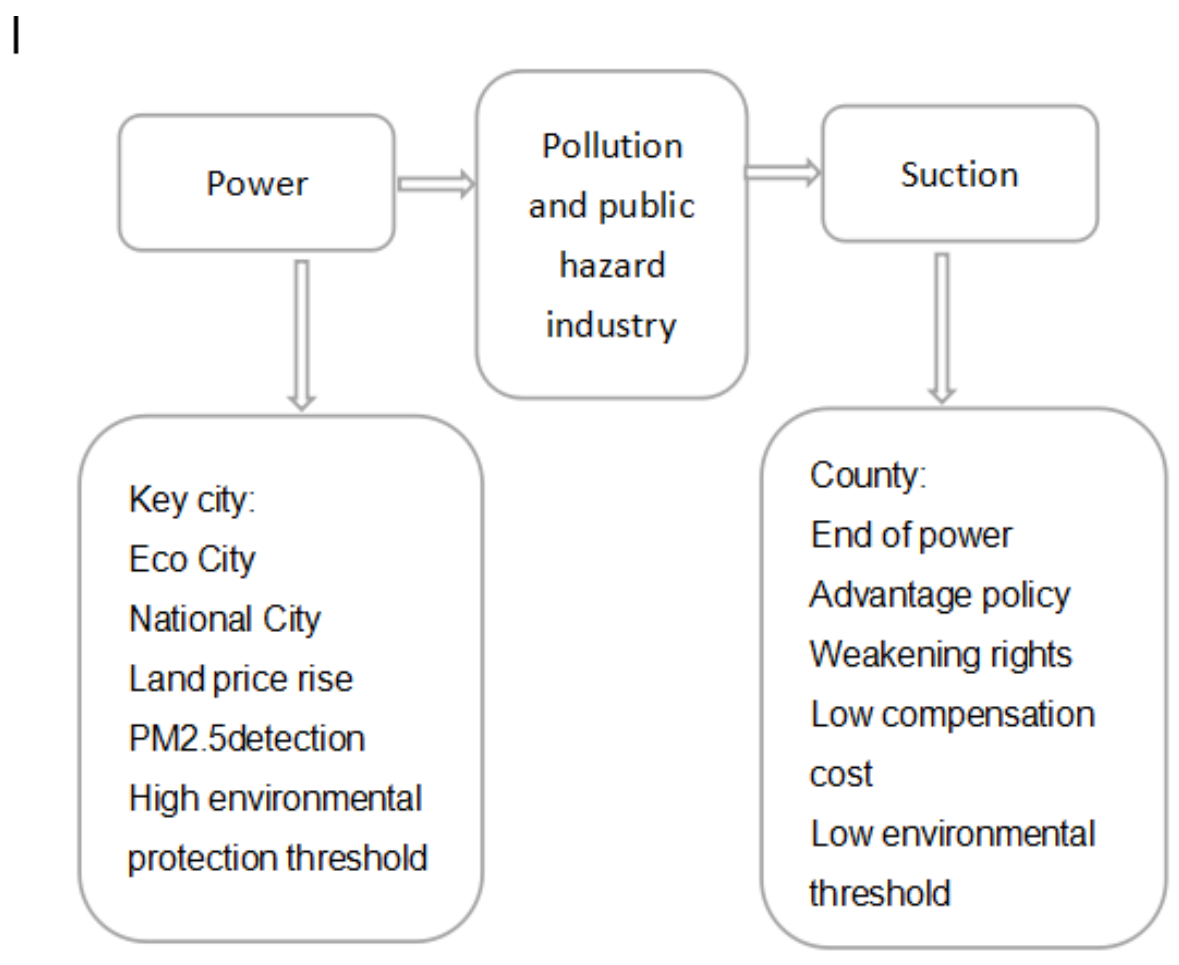

Figure 2 Schematic diagram of the dynamic mechanism of the transfer of pollution and pollution to the county

\section{The environmental injustice in the process of urbanization and its analysis}

2.1 Environmental injustice in the process of urbanization

2.1.1 Unfair distribution between human beings 
In the study of environmental sociology, environmental justice has two meanings: one means that everyone has the right to enjoy a clean environment, and the other is that the responsibility for environmental damage should be commensurate with the obligation of environmental protection. Environmental justice is concerned with issues in the environmental field. This is a problem that is of great importance to the future of mankind in China and the world at present. Some scholars believe that in the issue of environmental equity between urban and rural areas, cities always occupy an absolute advantage and rural areas an absolute disadvantage. They believe that no matter in production or life, cities have the problem of unconditionally transferring pollution to the countryside.

As the object of the fair distribution of environment, environmental goods and environmental evils are the core of the study of environmental justice. Environmental goods and environmental evils(as well as the environmental benefits and environmental burdens as their impacts) respectively refer to "any environmental characteristics that can be evaluated positively or negatively". Environmental goods usually refer to the environmental elements with positive value, while environmental evils are the environmental elements that harm people's survival.

\section{1) Unfair distribution of environmental goods}

Whether it is a higher environmental governance goal, or a more rigorous and effective environmental regulation means, let alone the introduction of environmental funds and the improvement of facilities, it is carried out in stages in China. There are significant differences in different regions, and the higher the administrative level of cities and regions, the more dominant in the distribution of environmental goods above. Small and medium-sized towns with relatively low administrative levels, for various reasons, not only have no priority, but even have no right to choose.

2) Unfair distribution of environmental evils

Looking at the whole process of social development, we can find that not all social strata will have the same damage to the environment. People with different social backgrounds and lifestyles have different impacts on the environment. At the same time, environmental risks are not evenly distributed to every member of society. Different social classes and different regions bear disproportionate environmental risks. Astypical environmental evils, the distribution of environmental pollution and environmental risks continues to shift to urban fringe areas. On the issue of environmental risks, the environmentally vulnerable groups, especially the low-income groups, often bear more environmental risks. Rural areas often bear more environmental pollution, but almost all the economic growth brought by environmental pollution is used for urban construction and urban pollution control.

\subsubsection{Environmental injustice between human and non-human species}

With the development of human industrial civilization and post-industrial civilization, the continuous expansion of cities, the decrease and disappearance of wilderness and pastoral, many wild animals have lost their original breeding habitats, extinct or become extinct; except for a few animalsdomesticated by humans;All but a few domesticated plants and animals are endangered. Even the species domesticated by humans, out of anthropocentrism, spent a brief and tragic life under cruel feeding methods, such as animals in modern cattle farms and chicken farms. Food from animals and plants, including meat at the expense of life, is stupidly wasted or used for trivial purposes.

\subsubsection{Injustice between humans and the entire ecosystem}

Humans are part of nature, the product of millions of years of evolution in the earth's ecosystem, and interdependent with everything else on earth. Since the industrial revolution, the whole ecological environment of the earth has been deteriorating rapidly. The greenhouse effect,ozone hole, the large-area water pollution, the air pollution, the soil pollution, the radioactive leakage and so on has seriously damaged many ecological balance of the whole earth.During urbanization, ecosystems are often damaged faster than they can be repaired. Both urban and rural areas are faced with rapid deterioration of the ecological environment. Since 2013, haze has often enveloped more than half of China. As the damage and feedback of human urban civilization to the ecological system, it has become the hottest topic of the environment.

\subsection{Consequences of unfair environmental distribution in the process of urbanization}

\subsubsection{The environmental damage in the county is particularly serious.}

With the transfer of high pollution enterprises to the county area, the emission of industrial "three wastes" is increasing. This will damage the air, water, soil and other ecological fields in the county area. The environmental pollution in the county is increasing, which seriously threatens the basic living conditions of the local people. According to official statistics, at present, "three wastes" polluted farmland has reached 0.1 billion hectares, 2.5 times more than 1983, and about $15 \%$ of farmland is polluted to different degrees.Over 4 million mu of farmland has been occupied and damaged due to the discharge of waste water and waste by highly polluting enterprises in China. $66 \%$ of rural drinking water meets the rural drinking water sanitation standards. More than 300 million rural people face unsafe drinking water problems, among which the harmful substances in drinking water of 190 million people exceeded the standard. 
In recent years, the air quality of cities above prefecture level has changed to a good trend, while the air quality of county-level cities has declined year by year, largely because of the consequences of the continuous transfer of pollution industry to the county. According to the data, the number of cancer villages in China exceeds 247, covering 27 provinces in mainland China. Excluding some cancer villages with unknown causes, the formation of the remaining $90 \%$ of cancer villages is related to modern industrial pollution. TakingDangjie village in Henan province as an example, the data from the 21st world anti cancer conference in August 2010 showed that the cancer incidence in China is in a rapid rise period, and the total cancer incidence is about $180 / 100000$, that is, 180 people in every 100000 people suffer from cancer. In less than three years, more than 20 people died of cancer in Dangjie village, which is nearly 100 times higher than the national incidence rate, and 5-6 cancer patients are newly added in the whole village every year. Qi River, seriously polluted by industry, is the only source of drinking water for villagers in Dangjie village.

\subsubsection{Frequent environmental crisis and environmental conflicts}

Mass incidents are the main manifestation of social conflicts in China, which is a prominent reflection of the accumulation of social contradictions. Group incidents refer to social conflicts caused by certain social contradictions, in which specific groups or unspecified majority (more than 10 people) jointly implement large-scale aggregation without legal basis, affect social order, endanger public security, and even cause casualties, public and private property damage. Environmental mass incidents refer to the group behaviors caused by environmental contradictions, involving some people and affecting enterprises and governments by means of collective petition, traffic jam, party and government organs and factories, etc., to protect their legitimate rights and interests infringed by environmental problems, with certain regional, scale, predictability, repeatability and harmfulness.

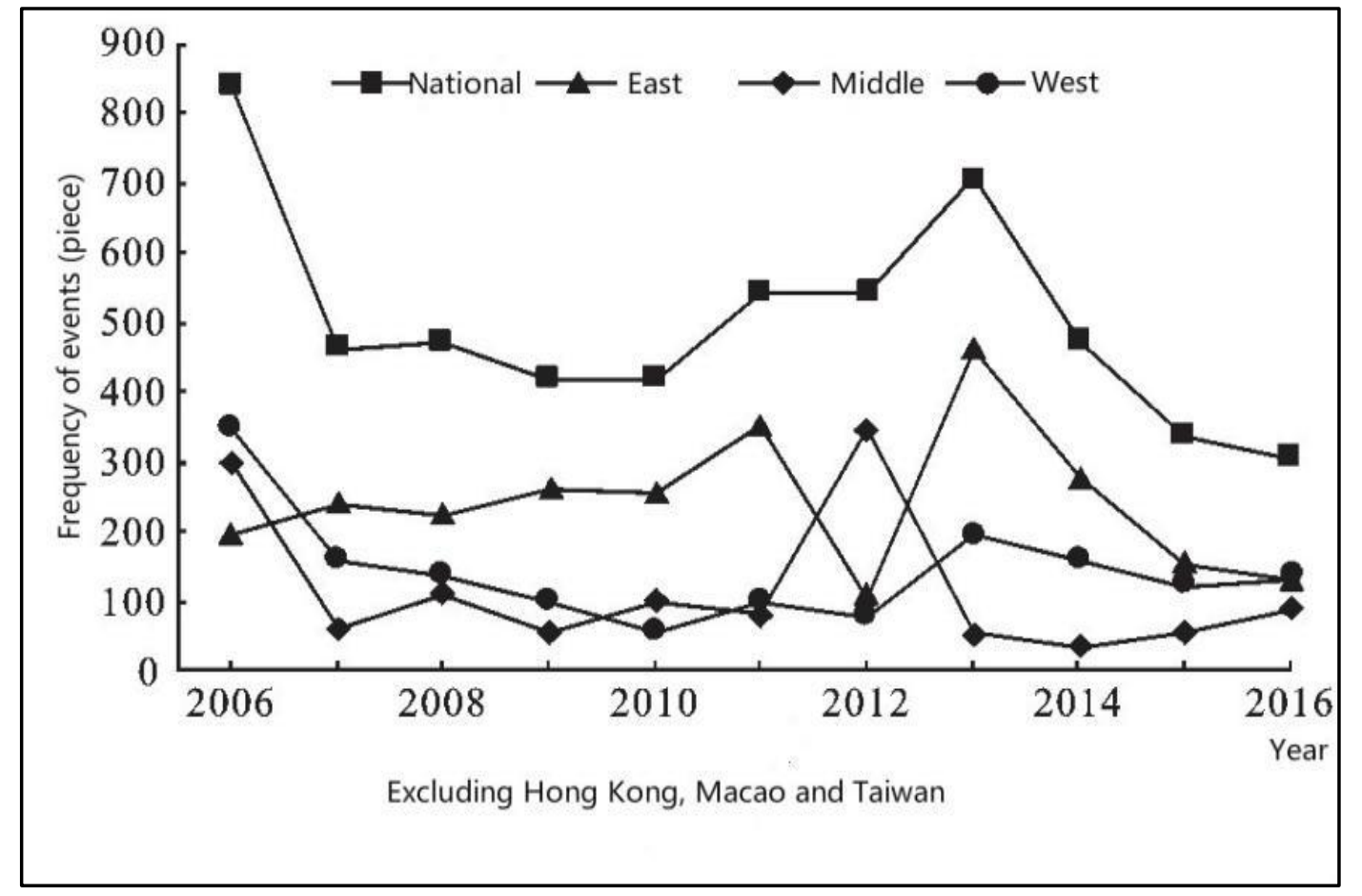

Figure 3 The change trend of environmental pollution incidents in China. (2006-2016) .

The data come from Spatio-temporal Distribution and Influencing Factors of Environmental Pollution Incidents Based on Multi-scales in China

It can be seen from the above figure that in recent years, due to the improper handling of the urbanization environmental governance issues, the environmental conflicts represented by the group events obviously exist in recent year.This reflects the current lack of environmental justice, especially the seriousness of the environmental governance dilemma and the need to solve the problem of environmental equity.

\subsection{Reasons for environmental injustice in the process of urbanization}

The reasons for environmental injustice are manifold.In the artificial ecosystem of cities, various ecological factors have extremely complex interactions in urban space; due to the shortage of human cognitive systems, the environmental effects or value facts of some new technologies, new materials, new industries and new projects are still unclear; The environmental value facts do not reach a consensus, and the environmental value objectives based on this cannot be unified; Some environmental facts are relatively clear, due to the social differences in politics, economy, 
culture and other aspects of environmental value subjects, low-income groups, small and medium-sized cities and rural residents as environmental subjects have not been sufficiently respected and recognized. In the social events such as urban demolition, land acquisition and relocation of polluting enterprises, there is an obvious phenomenon of being subject. And in the game of social subjects, due to the failure of the principal-agent mechanism, they are in a relatively unfavorable position and become vulnerable groups of the environment; Under the existing system of rules, procedural justice and distributive justice cannot be guaranteed, and environmental vulnerable groups are often ignored in the distribution of environmental value, leading to various social disputes.

\section{The construction of environmental justice system in the process of urbanization}

\subsection{Establishment of environmental value facts}

With the rapid development of science and technology, various new technologies are applied and promoted, and they are rapidly transformed into new industries. Numerous new materials and new products are put into the environmental system. According to statistics, there are now as many as 7 million known chemicals in the world, and the number of chemicals entering the environment has reached 100,000, all of which have never existed on the earth and are difficult to be degraded naturally. Scientists clearly admit that they are ignorant of a series of environmental effects, which contain huge environmental risks.

From the perspective of the history of science and technology and the history of environment, there is a long or short time lag between the emergence of environmental negative effects of a new technology and the solution of related problems. Once some serious environmental damage is caused, it is difficult to recover. For example, DDT caused the extinction of insects, fish and birds mentioned in "Silent Spring". The problem of white pollution caused by plastic bags in our country has been difficult to completely eradicate after many efforts; the PX projects that have caused mass incidents in various places in recent years are harmful or harmless? Should it be moved? Where should it move? There are different opinions. In today's scientific specialization, the majority of the non- professional public is in the position of information asymmetry. There is no unified view between the mainstream media and the official institutions, and there are different arguments in the academic community.

\subsection{Unification of environmental value goals}

The unity of environmental value goals has a significant impact on the construction of environmental justice system, which determines whether the residents in various regions have the same attention and treatment on environmental rights. The higher environmental value target means that the efficiency of environmental governance in this region will be greatly improved and the effectiveness of governance will be greatly improved. Although the environmental problem is a holistic one, the limited capital and manpower cannot guarantee the environmental governance in the direction of optimization. Therefore, the overall improvement of the environment and the key governance measures in some areas are taken. Therefore, the environmental goals are staged and hierarchical. Taking the treatment of PM2.5 as an example, with the same serious haze pollution, cities in Hebei province cannot get the same resources as Beijing, and the south-central cities belt of Liaoning province rank at the end of the phased governance plan published by the state.

The differentiation of environmental goals is mainly reflected in the differentiation of environmental goals between urban and rural areas and between regions. With the acceleration of economic development and the change of mode, the environmental problems in rural areas have become the key issues that must be properly handled in social development as urban areas. However, as cities occupy more favorable political and economic resources, the setting of environmental goals in urban areas is significantly higher than that in rural areas. The difference of environmental goals between regions is mainly manifested in that the environmental goals of the central cities in the metropolitan area are significantly higher than those of the surrounding cities. The underlying rules do exist, though there is no specific provision to prove it. Most of the people in the front-line central areas are economically and politically dominant groups, who have higher requirements for the environment and are able to put more pressure on pollution control and promote the migration of pollution industries.

\subsection{The game of environmental value subjects}

Referring to the experience of all over the world, the process of solving environmental problems and the institutional process of environmental justice system is also a dynamic process in which the forces of various environmental subjects are constantly changing and playing game. Environmental organizations and environmental movements in Europe and the United States have grown from nothing to growing up and exerting their influence in politics. Due to the improvement of the overall environmental awareness of the people in Taiwan province, the polluting industry has been unable to move freely within the island of Taiwan. At this stage, the transfer routes of the polluting industries in Taiwan province are mostly transferred to the mainland in China and Southeast Asia. 
Taiwan Six Light Industrial Park is the largest chemical park in the world. When the petrochemical industry expanded, the Formosa Plastic Group set its site on the east coast of the Taiwan strait, adjacent to the great cities Wetlands. This area is a rural area and the villagers have weak environmental awareness. The expansion of the petrochemical industry has long threatened the existence of the great cities wetlands and the oysters that grow in them, so environmental groups in Taiwan province persuaded local residents to fight together, which finally put an end to the golden age of Taiwan's petrochemical industry. The petrochemical industry, which has lost the opportunity of expansion in Taiwan, naturally aims at the mainland in China, especially Fujian province, which has become an ideal area for Taiwan's pollution industry transfer.

At present, the environmental non-governmental organizations in mainland China are developing, becoming the spokesperson of the vulnerable groups of the environment, and playing an increasingly important role in promoting the upgrading of environmental protection objectives and preventing environmental rights and interests damage. With the introduction and continuous improvement of environmental public interest litigation law, environmental accountability system and other systems, the game rules are in the process of development and change, and the overall approach to justice.

\subsection{Coordination of environmental value distribution}

From the traditional meaning of value, environmental value refers to the meaning of any thing to people's environmental nature and environmental needs, including human behavior. ${ }^{[\mathrm{i}]}$ The fulfillment of the environmental needs of any thing is embodied in the distributive justice between the environmental goods and the environmental evils, which is the symbol of the realization of environmental justice.

Environmental goods and environmental evils are an objective existence of the subject's cognitive scale. There is a serious lack of fair distribution within cities, between cities at all levels and between urban and rural areas. Environmental goods gather in central cities and central urban areas, while urban low-income groups, small and medium-sized urban residents, and rural residents involuntarily undertake a large number of environmental evils.

In the distribution of environmental values, environmental responsibility and environmental benefits should be unified, and environmental losses should be unified with environmental compensation. Some things tend to have both environmental goods and environmental evils attributes, such as green space, such as waste incineration plant, for living in different spaces of different subjects, there are completely different imagination. On the basis of fair game, the only way to eliminate environmental conflicts is to coordinate the interests of social subjects from all aspects and achieve fair distribution of environmental value.

\section{Concrete measures to promote the realization of environmental justice in the process of urbanization}

\subsection{Implement level: realize fair distribution of environmental infrastructure, etc.}

Environmental infrastructure, such as sewage treatment plants and landfill sites, should be distributed evenly between urban and rural areas. Selection of Sensitive site should be made to equitably solve the problems of water pollution, soil pollution and air pollution in urban and rural areas, so as to ensure the basic survival of the people.

The location of sensitive facilities should be fair, such as waste incineration center, high voltage lines and TV signal transmission towers and so on.Pollution industries should be treated ecologically and take the road of technology ecologicalization instead of transferring and spreading along the path of "least resistance and lowest cost".

In the reconstruction of urban and rural construction, we should adhere to the ecological principle, try our best to preserve the original historical and cultural landscapes, reserve breeding and habitat channels for wild animals and plants, and preserve biological diversity.

\subsection{Institutional level: further improve environmental responsibility and other laws and regulations}

The urban and rural environmental goals are basically the same, and the environmental construction planningsare basically synchronized. For the environmental damage that has been caused, we should actively repair the natural environment, make fair compensation to the victims, share the responsibility equitably, and pursue the responsibilities of those relevant responsible person, so that they will have no place to escape. For the project to be built, the environmental risks must be evaluated; If the evaluation is inconsistent with the later facts, the pursuit of responsibility is not a joke. We should actively guide the orderly development of the environmental movement and not make people live in fear.We will further improve the system of national nature reserves and national parks, and strictly implement the protection law on wetlands. While rationally exploiting natural resources, we should maintain ecological balance, restore the ecological environment and protect rare wild animals and plants.

4.3 Cultural level: Inheriting and developing social culture with environmental justice 
There are many ideas in Chinese cultural traditions that coincide with today's non-anthropocentrism, such as "Harmony between man and nature", "All people are my brothers and sisters, and all things are my companions.", "Don't do to others what you don't want others to do to you. ", "Axes enter the forest in time", etc. They are internationally recognized as one of the ideological sources of solving ecological environment problems. From the original meaning of these traditional words, their dissemination and understanding help people living in modern and post-modern times to equally treat nature, treat life and treat others.

There are also many critiques of environmental injustice in our cultural traditions, such as Mencius said that "the stable has fat horses, while the people have hunger.", and Baijuyi's poem "light fat" said that "the food is full and the heart is at ease, and the wine is full and the spirit is invigorating. It's a drought in the south of the Yangtze River. People in Quzhou eat people! " and so on. It's the same way that today's conscientious scholars criticize developed countries for wasting a lot of food, but watch hundreds of millions of people around the world fall into malnutrition, which is an important manifestation of environmental injustice. We should vigorously inherit and develop the idea of environmental justice in traditional culture, combine it with the Western non-anthropocentrism, connect with Chinese practice and the problems that need to be solved most, and establish a social culture of environmental justice with Chinese characteristics.

\section{References}

ChenTianpeng.(2008). Study on Eco-city Construction and Evaluation .Harbin, Heilongjiang: Harbin University of Technology,11-14.

Chen Min.(2009). Research on Pollution Transfer in Industrial Gradient Transfer .Hangzhou, Zhejiang: Zhejiang University, 30-31.

Li Wenjia.(2009), Analysis of Rural Transfer of High Pollution Enterprises,ModernCommercial Industry, (10): 82-83.

Li Deshun.(2007).Theory of Value (Second Edition), Beijing: Renmin University of China Press, 445.

Oriental hotline.(2010-12-20). Direct attack: map of "cancer village" in China [EB / OL]. .Http://news.cnool.net/0-18/24252/5.html.

WangHongru.(2005). Relocation of ShougangGroup to the East - tax Distribution and Pollution Transfer Become TheFocus of Resettlement. China Economic Weekly, (3): 21.

Wang Taoyang. David Miller. (2012).On Environment Goods.Philosophical Trends. (10): 84-90.

Wang Yong.(2012-04-11).The Incidence of 20 Deaths in a Cancer Village in Henan Province in ThreeYearsis 100 Times Higher Than That in China [EB / OL]. .http://news.qq.com/a/20120411/000608_2.htm.

Wang Yuming.(2012).Analysis of The Causes of Violent Environmental Group Events -- Based on the Study of Ten Typical Environmental Group Events. Journal of Zhuhai University of Administration,(3): 37-42.

XieYufeng,MaXiaoming.(2007).A Summary of the Studies on Current Situation of Environmental Equity in China. Ecological Environment.(2): 393.

Zhang Feinan.(2010).Countermeasures to Promote Urban and Rural Environmental Equity.Journal of Suihua University, (6): 68-69.

Zhao Jincheng.(2012). Study on TheCauses of Rural Industrialization and Environmental Pollution. Forum on Industry and Science and Technology,(11): 227-227.

Zhao Xueyan, Wang Rong, Wang Xiaoqi, Liu Jianghua.(2019).Spatio-temporal Distribution and Influencing Factors of Environmental Pollution Incidents Based on Multi-scales in China.Geosciences, 39 (09): 1361-1370.

Zhang Yuandong.(2012). Study on Classification and Classification Management of Chemical Environmental RisksSources. Shandong Chemical Industry,(2): 81-83. 